\title{
Bioética y alimentación al final de la vida en pediatría: dos perspectivas complementarias sobre un mismo tema
}

\author{
Bioethics and end of life feeding in pediatrics: Two complementary \\ perspectives about one single topic \\ Bioética e alimentação no final da vida em pediatria: duas perspectivas complementares \\ sobre o mesmo tema
}

Jorge Jacobo Arenas-Rivera' ${ }^{1}$ Javier Portilla-Segura², Evelia Apolinar-Jiménez ${ }^{3 *}$.

Recibido: 7 de julio de 2021. Aceptado para publicación: 31 de agosto de 2021. https://doi.org/10.35454/rncm.v4supl1.324

\begin{abstract}
Resumen
Con los avances en las terapias curativas se espera que los niños, aun enfermos, sobrevivan a sus padres; sin embargo, existen condiciones imposibles de curar, que limitan la esperanza de vida. Los cuidados paliativos pediátricos se refieren al cuidado activo y total del cuerpo, mente y espíritu de los niños, e incluyen soporte a su familia. La alimentación e hidratación artificial pueden ser una alternativa al final de la vida que contribuya con el control de los síntomas; estas tienen connotaciones psicológicas, culturales, religiosas $y$, por tanto, bioéticas. Es necesaria la reflexión, pues además de contar con conocimientos técnicos y científicos, la atención de niños y adolescentes con enfermedades imposibles de curar implica la comprensión integral de principios y valores éticos. Esta revisión pretende invitar a la reflexión al abordar el tema de la muerte y del papel de la alimentación al final de la vida en pediatría, desde las perspectivas de la bioética y de la práctica clínica. A partir de la perspectiva bioética se detallan los principios de beneficencia, autonomía, justicia y no maleficencia, mismos que en la atención de menores enfermos en fase terminal se deben potenciar con la benevolencia, la autonomía asistida, la magnanimidad y la no malevolencia. Estos conceptos permitirán ver, desde la óptica
\end{abstract}

\section{Summary}

With advances in curative therapies, children, even if ill, are expected to outlive their parents. However, there are impossible-to-cure conditions that limit life expectancy. Pediatric Palliative Care refers to the active and total care of the body, mind and spirit of children, including support for their families. Artificial feeding and hydration can be an alternative at the end of life to help control symptoms, albeit with psychological, cultural, religious, and bioethical connotations. Reflection is necessary, because the care of children and adolescents with illnesses that cannot be cured is not only a matter of technical and scientific knowledge but also of a comprehensive understanding of ethical principles and values. This review aims to invite reflection regarding the process of dying and the role of feeding at the end of life in pediatric patients, from both the bioethical and clinical practice perspectives. From the bioethical perspective, the principles of beneficence, autonomy, justice, and no-maleficence are detailed, which in the care of terminally ill children must be strengthened with benevolence, assisted autonomy, magnanimity, and nomalevolence. These concepts will allow us to see, from the bioethical perspective, the transcendence of practical decisions, the subtle difference between trying nutritio-

\section{Resumo}

Com os avanços nas terapias curativas, espera-se que as crianças, mesmo doentes, sobrevivam aos pais. No entanto, existem condições impossíveis de curar que limitam a expectativa de vida. Os Cuidados Paliativos Pediátricos referem-se ao cuidado ativo e total do corpo, mente e espírito das crianças, incluindo o apoio a sua família. A alimentação e a hidratação artificiais podem ser uma alternativa no final da vida que contribui para o controle dos sintomas, pois tem conotações psicológicas, culturais, religiosas e, portanto, bioéticas. A reflexão é necessária, pois, além de possuir os conhecimentos técnico-científicos, a atenção das crianças e dos adolescentes com enfermidades incuráveis implica a compreensão integral dos princípios e valores éticos. Esta revisão tem como objetivo convidar à reflexão ao abordar a questão do morrer e o papel da alimentação no final da vida em pediatria, sob as perspectivas da bioética e da prática clínica. Do ponto de vista bioético, detalham-se os princípios da beneficência, autonomia, justiça e não maleficência, os mesmos que na atenção ao menor em estado terminal, devem ser fortalecidos com a benevolência, a autonomia assistida, a magnanimidade e a não malevolência. Esses conceitos permitirão ver, do ponto de vista bioético, a transcen- 
bioética, la trascendencia de las decisiones prácticas, esa sutil diferencia entre intentar el soporte nutricional, que limita el sufrimiento y busca mejorar la calidad de vida, y promover terapias fútiles, que pueden alargar la agonía y el sufrimiento, incrementar el desgaste económico y generar falsas expectativas.

Palabras clave: bioética, alimentación al final de la vida, niños, adolescentes, calidad de vida.

Comité Hospitalario de Bioética, Clínica Bajío CLINBA, SC. Guanajuato Guanajuato, México.

2 Servicio de Medicina del Dolor y Cuidados Paliativos, Hospital General Regional de León. León, Guanajuato, México.

\section{INTRODUCCIÓN}

Con los avances científicos y tecnológicos de la medicina moderna han aumentado significativamente las terapias curativas para múltiples enfermedades propias de la edad pediátrica. De tal modo que en la actualidad se espera que, en general, los hijos sobrevivan a sus padres $^{(1)}$. Sin embargo, hay condiciones congénitas o adquiridas en la infancia que no se pueden curar y limitan la esperanza de vida ${ }^{(2)}$. En estas situaciones es indispensable ofrecer los mejores cuidados que permitan acompañar a los pacientes y a sus familias, así como a los profesionales de la salud que proveen estas atenciones. Es por ello que los cuidados paliativos pediátricos (CPP) han tomado relevancia, especialmente en las últimas dos décadas. Según la Organización Mundial de la Salud (OMS), su objetivo es prevenir y aliviar el sufrimiento físico, psicológico, social y espiritual, y esto es, además, una responsabilidad ética global ${ }^{(3)}$.

El papel de la intervención nutricional y de la alimentación al final de la vida, además de ser una de las herramientas que puede contribuir al control de síntomas ${ }^{(4)}$, tiene profundas connotaciones psicológicas, culturales, religiosas y, por tanto, éticas ${ }^{(5)}$.

Las causas de muerte son esencialmente diferentes en la edad pediátrica y en la adulta, por lo que el abordaje de los CPP es también diferente entre estos dos grupos etarios ${ }^{(1,2)}$. Nosotros, como profesionales de la salud que atendemos pacientes pediátricos con enfermedades complejas e imposibles de curar, con frecuencia dência das decisões práticas, aquela sutil diferença entre experimentar um suporte nutricional que limite o sofrimento e procure melhorar a qualidade de vida ou promover terapias fúteis que podem prolongar a agonia, prolongar o sofrimento, aumentar o desgaste econômico e gerar falsas expectativas.

Palavras chave: bioética, alimentação no final da vida, crianças, adolescentes, qualidade de vida.

\author{
3 Unidad de Metabolismo y Nutrición, Departamento de Investigación \\ Hospital Regional de Alta Especialidad del Bajío. León, Guanajuato, \\ México. \\ *Correspondencia: Evelia Apolinar-Jiménez. \\ eve.apolinar@gmail.com
}

seguimos enfrentando la muerte de un menor de edad como algo antinatural, con dificultad para elegir el mejor momento en el que un niño o adolescente se beneficiará al recibir CPP y evitar tratamientos que pueden ser inútiles, invasivos y costosos ${ }^{(6)}$. Es un imperativo ético contar con elementos tanto teóricos como técnicos que ayuden a evitar el sufrimiento en los pacientes y sus familias y, cuando inexorablemente se presenta, paliarlo.

Esta revisión pretende abordar el tema de la muerte y del papel de la alimentación al final de la vida en pacientes pediátricos desde las perspectivas filosófica y bioética, y de los profesionales sanitarios, y con ello contribuir a la reflexión que permita, como lo anota Bergstraesser $^{(2)}$, redirigir el significado de la esperanza en este último período de la vida.

\section{BIOÉTICA Y ALIMENTACIÓN AL FINAL DE LA VIDA EN PEDIATRÍA. DOS PERSPECTIVAS COMPLEMENTARIAS SOBRE UN MISMO TEMA}

\section{Perspectiva bioética: de los derechos humanos a la reflexión sobre la muerte en los menores de edad}

«LLUVIA EN COPACABANA. Como cae la lluvia sobre el mar / al ritmo que sin pausa se desploma / así vamos fluyendo hacia la muerte.». Esta reflexión, del poeta mexicano José Emilio Pacheco( ${ }^{(7)}$, dice una verdad sabida por todos los seres humanos de todos los 
tiempos: es inevitable morir. Muchos sentimientos se pueden tener ante la muerte, entre ellos, es común que se produzcan el temor y la tristeza. Temor ya que se le rehúye a la muerte por ser algo «dañino», por cierto, lo más dañino que le puede suceder a cualquier ser vivo: dejar de ser; tal sentimiento invade esencialmente al que va a morir. Y la tristeza, pues ser consciente de que se va a dejar de vivir causa un profundo pesar y mucho abatimiento. Este sentimiento no solo lo experimenta quien está por morir, sino también las personas cercanas a quien agoniza. La muerte, en mayor o menor medida, implica, entre otros muchos sentimientos, temor y tristeza. Estos se acrecientan cuando quien está a punto de morir es un niño o adolescente que, además, padece una enfermedad grave, la cual ya no es posible curar. Como señala el filósofo alemán Martin Heidegger, «Tan pronto como un hombre entra en la vida es ya bastante viejo para morir ${ }^{\left({ }^{8}\right)}$; la muerte de un menor no deja de causar gran temor y tristeza, y puede llegar a convertirse en tragedia. Lo es, primeramente, porque en la «lógica» de la vida, esta se le acorta a un ser humano que por edad tenía aún mucho por vivir y realizar. Además, porque ese proceso de enfermedad provoca, a quien la padece y a los suyos, dolores físicos $y$ emocionales de profunda intensidad.

Ante situaciones dolorosas, como la señalada, se debe buscar el mejor escenario, este debe ser procurar una buena vida por el tiempo que le resta al menor que padece una enfermedad en etapa terminal, para que todo esto desemboque en una buena muerte. Pero esto no se queda a nivel del puro desear lo bueno para los enfermos en tales condiciones; ese buen vivir, y un consecuente buen morir, es un derecho humano; por tanto, la vida, en específico la buena vida, es algo que cualquier ser humano puede exigir. Así ha quedado consagrado en el Artículo 3 de la Declaración Universal de los Derechos Humanos de la Organización de las Naciones Unidas (ONU): «Todo individuo tiene derecho a la vida, a la libertad y a la seguridad de su persona ${ }^{(9)}$.

Ahora bien, en cuanto derecho, para ser «justiciable» este artículo 3 de la citada Declaración, es decir, para darle realidad y que no quede en mera promulgación, es el Estado el principal garante de ello, pues solo él tiene la fuerza legal y material para garantizarlo. Sin embargo, todos seres humanos debemos promover el cumplimiento de dicho artículo, seamos parte o no del Estado (entendido este como gobierno), ya que los derechos humanos están destinados para beneficio de todos. No obstante, no todas las personas estamos obligadas a darle realidad a dicho artículo de igual manera, sino conforme a las condiciones y circunstancias de cada uno, como lo señala el principio de justicia: «Distribución equitativa de los recursos, beneficios y cargas entre todos los miembros de la sociedad $\gg^{(10)}$.

Dentro de los miembros de la sociedad que aportan mucho en la promoción del derecho a una buena vida de los niños y adolescentes enfermos graves que no podrán curarse están los profesionales de la salud. Esto es así ya que estos profesionales cuentan con la autoridad epistémica, que les da sus conocimientos, para hacer posible, en gran medida, una buena vida y una buena muerte de estos enfermos. Esos conocimientos son imprescindibles, pero insuficientes si se quiere atender de la manera más humana posible a estos pacientes en situación crítica y en fase terminal. También habrá que tener en cuenta ciertas pautas éticas, que al atenderlas promocionen una atención sensible, empática y lo más éticamente humana.

Aquí se proponen las siguientes pautas éticas. Primero, tener en consideración que un menor, niño $o$ adolescente que padece alguna enfermedad imposible de curar, la cual deteriora la calidad de vida, es un sujeto vulnerable, esto es una persona que por alguna condición o situación específica es incapaz o está impedida para ejercer de manera plena su autonomía ${ }^{(11)}$. La vulnerabilidad radica en el hecho de que, por su corta edad, aún no se tiene la madurez cognitiva para comprender a total cabalidad su situación y, a partir de esto, tomar las decisiones más razonables. Dicha madurez se va acrecentando en la medida que los pacientes van obteniendo la capacidad de realizar procesos cognitivos más complejos, lo cual supone una madurez biopsíquica, que se da con el tiempo, a no ser que padezca alguna alteración neurológica.

Consecuencia de esta primera pauta ética puede recomendarse tener en cuenta las orientaciones éticas propuestas en los ya célebres cuatro principios bioéticos de beneficencia, autonomía, justicia y no maleficencia, pero no atendidos sin más como tales, sino potenciándolos con otras consideraciones éticas, que exigen la atención lo más humanizada posible de esos sujetos vulnerables que son los niños y adolescentes con enfermedad grave en fase terminal; estos son:

- Principio de beneficencia: «Hacer bien una actividad y hacer el bien a otros mediante una actividad bien hecha» ${ }^{(12)}$. En efecto, el personal de la salud debe ofrecer bienes físicos y emocionales al menor enfermo, a través de un buen diagnóstico, buscar los medicamentos y procedimientos clínicos más idóneos, con la intención de obtener el máximo 
beneficio terapéutico, entre otros. Este principio se potencia aún más si lo respalda la benevolencia, esa intención ética que pretende lograr el bien del otro. En efecto, tiene más posibilidad de lograr el mayor beneficio aquel que no es indiferente al paciente $y$ que a la vez está motivado por querer el bien (benevolencia) de su paciente, pues esa intención será un buen impulso extra para buscar la beneficencia.

- Principio de autonomía: «Respeto activo a la libertad de elección de la persona; no solo absteniéndose de obstaculizarla, sino también procurando que se den las condiciones necesarias para ejercerla ${ }^{(10)}$. En atención a lo ya dicho, el niño o adolescente es un sujeto vulnerable, pues por su inmadurez cognitiva y emocional a causa de su edad, no puede ejercer de manera plena su autonomía, así que hay que tener en cuenta una autonomía asistida; esto se refiere a padres o tutores que han de tomar las decisiones en beneficio del enfermo que tutelan, a partir de una información completa, veraz y entendible en sus términos. Aquí el personal de salud ha de esforzarse para que se logren esos rasgos de una buena comunicación, para que padres o tutores tomen decisiones a partir de datos veraces, fiables y entendibles. Asimismo, no está de más señalar que si las condiciones de salud física, emocional y la madurez cognitiva ya lo permiten, el menor también deberá dar su aprobación sobre las terapias y procedimientos que se le sugieran.

- Principio de justicia: «Distribución equitativa de los recursos, beneficios y cargas entre todos los miembros de la sociedad» ${ }^{(10)}$. Aunque lo mínimo que se le puede pedir al personal de la salud (como a cualquier otro profesional) es que cumpla lo que por ley se le pide, la vulnerabilidad en que se encuentra un niño enfermo exige éticamente algo más que la sola justicia, esto es la magnanimidad. Esta es esa virtud que consiste en dar más de lo que está establecido por ley (lo justo), sin fijarse en pequeñeces. Si se practica esta virtud algunas veces, el profesional de la salud se quedará a consultar a sus pacientes, incluso un poco después de su horario, o habrá algunas noches en que se desvelará revisando literatura clínica para ahondar sobre la patología que presente alguno de sus pacientes. Este tipo de profesionales son los que hacen la buena diferencia para el paciente y sus cercanos, así como para sí mismos, pues estas acciones los hacen crecer éticamente.

- Principio de no maleficencia: «Ante todo, no hacer daño» ${ }^{(12)}$. Al igual que en el principio de beneficencia, pero de manera inversa, el no hacer el mal (el mínimo exigible para cualquier profesional) se potencia más si se hace de lado cualquier intención de querer hacer daño (malevolencia). Sin embargo, hay una sutil, pero profunda diferencia entre cumplir la beneficencia y la no maleficencia: éticamente no estamos obligados a causar un bien, pero sí a no provocar un mal. Esto es, por ejemplo, que no se está obligado a quedarse más allá del horario de trabajo para atender a un niño enfermo o resolver una duda a sus padres, pero lo que no está permitido, en términos éticos, es no atenderles cuando se esté en el horario que se destine para ello.

Retomando los sentimientos de temor y tristeza que acompañan a los menores enfermos y a sus padres, se han sugerido algunas pautas de cómo se podría actuar éticamente con ellos para mitigar ese sufrimiento a partir de las actitudes de quienes acompañan a estos pacientes en su enfermedad, en específico, cómo podrían proceder los profesionales de la salud, además de todo el conocimiento técnico y científico indispensable para orientar a los padres y los menores, y así tomar las mejores decisiones de tratamiento. Con los pacientes en una condición tan doliente, tal vez ya no se logre dar grandes placeres, pero al menos sí se les pueden evitar sumos dolores en esas horas de profundo sufrimiento. Algo muy importante es que, en esa situación lacerante, y en la cual la muerte es inminente, paliar el dolor es algo de incalculable valor, no solo para la persona directamente afectada, sino también para sus cercanos, pues como señalaba Epicuro, filósofo de la antigua Grecia, la felicidad consiste (según lo cita el doxógrafo Diógenes Laercio) en obtener el placer y, si esto no es posible, ya se logra mucho evitando el dolor ${ }^{(13)}$.

\section{Perspectiva sanitaria y de los profesionales de la salud}

\section{El trabajo multidisciplinario en los equipos de CPP}

Los niños que cursan con condiciones crónicas complejas requieren un manejo especializado por diferentes profesionales, que puedan centrarse en la atención del paciente y su familia ${ }^{(14)}$, es por ello que existen los CPP, los cuales se refieren al cuidado activo y total del cuerpo, mente y espíritu de los niños, dando soporte a su familia ${ }^{(15)}$. A diferencia del abordaje de los cuidados paliativos en los adultos, en CPP existen 4 grupos de atención ${ }^{(16,17)}$ :

- Grupo 1: niños en situaciones de amenaza para la vida y en los que el tratamiento curativo es posible (cáncer, cardiopatías congénitas, falla orgánica); 
- Grupo 2: niños en situación de muerte prematura inevitable, en quienes el tratamiento puede prolongar la vida (fibrosis quística);

- Grupo 3: niños con progresión de su enfermedad, sin opciones de tratamiento curativo (errores congénitos del metabolismo, enfermedades neuromusculares);

- Grupo 4: condiciones irreversibles que pueden causar discapacidades; cursan con alteraciones neurológicas que pueden complicar el estado de salud y con probabilidad de que ocurra una muerte prematura (parálisis cerebral grave).

En caso de pacientes pediátricos con enfermedades crónicas complejas (ECC), independientemente del diagnóstico, es importante identificar el grupo en el que se encuentran para dirigir de manera óptima la intervención del equipo multidisciplinario de CPP, cuyo objetivo en el niño y su familia es mantener la calidad de vida del paciente ${ }^{(18)}$, el cual puede cursar con fluctuaciones, considerando la enfermedad, etapa clínica y edad del paciente.

Además, es necesario señalar que según la American Academy of Pediatrics, los profesionales de la salud deben contar con el apoyo del equipo de cuidados paliativos para enfrentar el proceso de agonía y muerte del niño ${ }^{(1)}$. No es raro que los profesionales de la salud que atienden pacientes pediátricos con ECC, especialmente quienes no forman parte de los equipos de CPP, se decanten por intervenciones fútiles, que no cambiarán el desenlace y que, en ocasiones, pueden alargar la agonía, prolongar el sufrimiento, generar falsas expectativas o incrementar el desgaste económico familiar. Entre los principales obstáculos de los CPP se describen la preocupación de médicos por quitar la esperanza, la incertidumbre sobre el pronóstico o no identificar el momento adecuado para modificar el abordaje ${ }^{(6)}$.

\section{EL PAPEL DE LA ALIMENTACIÓN AL FINAL DE LA VIDA}

Comer es una práctica familiar importante. Es principalmente dentro de las familias donde se adquieren y experimentan las preferencias alimentarias, así como los comportamientos relacionados con la alimentación y las identidades. También es donde aprendemos a disfrutar (aunque no siempre) de la experiencia corporal de la comida (cómo sabe, huele y se siente), sus aspectos materiales, sensoriales y afectivos. La alimentación tiene una importancia social, simbólica para las familias que tienen un enfermo terminal ${ }^{(19)}$.
La alimentación en personas enfermas es una de las preocupaciones más importantes entre su familia, pues dar de comer y de beber son símbolos de cuidado ${ }^{(20,21)}$, especialmente si el enfermo es un menor ${ }^{(22)}$. La intervención nutricional y la alimentación en niños con enfermedades crónicas e incurables se puede evaluar desde varias perspectivas. Tal es el caso de los niños que padecen deterioro neurológico grave, en quienes la alimentación artificial, mediante sondas enterales, puede tener un impacto discreto en la calidad de vida, pero puede allanar el trabajo de padres y cuidadores al facilitar la alimentación y la administración de medicamentos $^{(23)}$. En general, mientras el paciente pueda y tenga deseos de comer y beber, aunque mejorar el peso o el estado nutricional no sea un factor para modificar el desenlace, debe mantenerse la alimentación ${ }^{(22,24)}$.

En las etapas avanzadas del padecimiento es común que los niños cursen con intolerancia a la vía oral, condicionada por el deterioro funcional, resultado de la enfermedad misma o de los tratamientos ${ }^{(22)}$. Se recomienda proveer alimentación e hidratación artificial cuando hay devastación neurológica, falla intestinal extensa e irreversible, y muerte próxima por cualquier causa $^{(22,25)}$. Los cambios en la alimentación pueden incluir nutrición por vía enteral o parenteral, o bien, hidratación artificial, administrada mediante dispositivos $^{(14,26)}$. Sin embargo, cuando las alteraciones en la alimentación ocurren, es necesario individualizar cada abordaje, ya que no siempre los dispositivos invasivos desempeñan un rol importante, por lo que se necesita definir el objetivo terapéutico.

No es raro que la decisión entre utilizar o no la nutrición y la hidratación artificial provoque opiniones diferentes entre los miembros del equipo de salud, los pacientes o sus familiares ${ }^{(20)}$. En estos casos es indispensable el diálogo y la comunicación con los padres $y$, si es prudente, con el escolar o adolescente enfermo, en términos sencillos, en un entorno sensible, solidario y sin prejuicios ${ }^{(22)}$; es decir, haciendo conscientes y ejerciendo los principios y valores éticos de los que se habló anteriormente.

\section{REDIRIGIR EL SIGNIFICADO DE LA ESPERANZA}

Hay una contradicción innata en los profesionales de la salud, en tanto seres humanos, entre el deseo de mejorar los CPP y la atención al final de la vida de los niños y sus familias, y la necesidad de protegerles de las potenciales cargas adversas y de las malas noticias ${ }^{(27)}$. 
Ello puede llevar a los profesionales de la salud a intentar medidas invasivas, que no cambiarán el curso de la enfermedad y su inevitable desenlace; además, en algunos casos no mejorarán la calidad de vida en este final del trayecto. Entre ellas, la alimentación (como símbolo de crianza y de vitalidad) y la nutrición e hidratación artificial pueden, en algunas ocasiones, generar falsas esperanzas, especialmente entre quienes anhelan en lo más profundo para su hijo amado enfermo que ocurra un milagro.

\section{CONCLUSIÓN}

Es importante tanto entre los equipos de CPP, como entre el resto del personal de salud que atiende a los niños y adolescentes que padecen enfermedades crónicas complejas e imposibles de curar desarrollar una comprensión más integral de cómo estos padecimientos son experimentados por los niños afectados y sus familias y, a su vez, diseñar intervenciones que eviten el sufrimiento y apoyen la calidad de vida ${ }^{(1,28)}$. Entonces, a partir de la conciencia bioética, se logra tener una actitud solidaria y empática, en suma, éticamente buena.

\section{PUNTOS CLAVE}

- Los niños y adolescentes con enfermedades crónicas complejas, que no tienen posibilidad de cura, requieren intervención de equipos multidisciplinarios que busquen controlar síntomas, evitar el sufrimiento físico y emocional, y mantener la calidad de vida lo mejor posible.

- Es un ideal en la práctica profesional que los principios bioéticos, beneficencia, autonomía, justicia y no maleficencia, aplicados a la atención y cuidado de los pacientes pediátricos con enfermedades terminales, se acompañen de la benevolencia, la autonomía asistida, la magnanimidad y la no malevolencia.

- La alimentación desempeña un rol social y simbólico importante en las familias. Hace referencia a la crianza y al cuidado de los más vulnerables: los niños y adolescentes enfermos.

- El papel de la alimentación e hidratación artificial al final de la vida puede, en algunos casos, limitar el sufrimiento y mejorar la calidad de vida.

- Es imprescindible la reflexión bioética, a fin de tener una práctica profesional solidaria y empática, es decir, éticamente buena.

\section{Fuente de financiación}

El presente estudio no tuvo financiación.

\section{Conflicto de intereses}

Los autores declaran no tener conflicto de intereses.

\section{Declaración de autoría}

J Arenas, J Portilla y E Apolinar contribuyeron igualmente a la concepción de la revisión; E Apolinar contribuyó al diseño de la revisión; J Arenas, J Portilla y E Apolinar redactaron el manuscrito. Todos los autores revisaron el manuscrito, acuerdan ser plenamente responsables de garantizar la integridad y precisión del trabajo, y leyeron y aprobaron el manuscrito final.

\section{Referencias bibliográficas}

1. American Academy of Pediatrics. Palliative care for children. Pediatrics. 2000;106(2):351-7. doi: 10.1542/peds.106.2.351.

2. Bergstraesser E. Pediatric palliative care - When quality of life becomes the main focus of treatment. Eur J Pediatr. 2013;172(2):139-50. doi: 10.1007/s00431-012-1710-z

3. Palliative care [Internet]. World Health Organization. Consultado el 3 de julio de 2021. Disponible en: https:// www.who.int/westernpacific/health-topics/palliative-care

4. Hui D, Dev R, Bruera E. The last days of life: Symptom burden and impact on nutrition and hydration in cancer patients. Curr Opin Support Palliat Care. 2015;9(4):346-54. doi: 10.1097/ SPC.0000000000000171

5. Apolinar-Jiménez E, Tena-Tamayo C, Allende-Pérez S, Portilla-Segura J. ¿Estamos atendiendo de manera oportuna a los pacientes con enfermedad terminal? El papel de los cuidados paliativos y la nutrición. Hosp Med Clin Manag. 2018;11:13-9. doi: 10.24875/HMCM.18000105

6. Hauer JM, Wolfe J. Supportive and palliative care of children with metabolic and neurological diseases. Curr Opin Support Palliat Care. 2014;8(3):296-302. doi: 10.1097/ SPC. 0000000000000063

7. Pacheco JE. Tarde o temprano (Poemas 1958 - 2000). 1. a edición. Fondo de Cultura Económica. 2004. p. 655.

8. Heidegger M. El ser y el tiempo. 2.a edición. México: Fondo de Cultura Económica. 1971.p. 152.

9. La Declaración Universal de Derechos Humanos [Internet]. Naciones Unidas [consultado el 1 de julio de 2021]. Disponible en: https://www.un.org/es/about-us/universaldeclaration-of-human-rights

10. Sánchez González MA. Bioética en ciencias de la salud. 1. ${ }^{a}$ edición. Barcelona, España: Elsevier Masson; 2013. p. 456. 
11. Gracia D. Procedimientos de decisión en ética clínica. $1 .^{a}$ edición. Madrid, España: Tricastela; 2007.p. 160.

12. Hortal A. Ética general de las profesiones. 1.a edición. Bilbao, España: Desclée de Brouwer; 2002. p. 278.

13. Laercio D. Vida de los filósofos más ilustres. 2004. p. 413.

14. Schwantes $S, O$ 'Brien HW. Pediatric palliative care for children with complex chronic medical conditions. Pediatr Clin North Am. 2014;61(4):797-821. doi: 10.1016/j.pcl.2014.04.011

15. What is children's palliative care? [Internet]. International Children's Palliative Care Network [consultado el 3 de julio de 2021]. Disponible en: https://www.icpcn.org/about-icpcn/ what-is-childrens-palliative-care/

16. Tirado-Perez IS, Zarate-Vergara AC. Clasificación de la Association for Children's Palliative Care (ACT) en una institución pediátrica de referencia en el Caribe colombiano. Rev Salud Pública. 2018;20(3):378-83. doi: 10.15446/rsap. v20n3.60797

17. Association for Children's Palliative Care. A guide to the development of children's palliative care services. Bristol, RU: Association for Children's Palliative Care (ACT); 2009.

18. Keele L, Keenan HT, Sheetz J, Bratton SL. Differences in characteristics of dying children who receive and do not receive palliative care. Pediatrics. 2013;132(1):72-8. doi: 10.1542/ peds.2013-0470

19. Ellis J. Family food practices: Relationships, materiality and the everyday at the end of life. Sociol Health Illn. 2018;40(2):353-65. doi: 10.1111/1467-9566.12606

20. Holmes S. Withholding or withdrawing nutrition at the end of life. Nurs Stand. 2010;25(14):43-6. doi: 10.7748/ ns2010.12.25.14.43.c8154

21. Allende-Pérez S, Pérez-Camargo DA, Itaí Urbalejo-Ceniceros V, Ochoa-Carrillo FJ. Evaluación del estado de nutrición en pacientes con cáncer avanzado, referidos a cuidados paliativos del Instituto Nacional de Cancerología, México. GAMO. 2013;12(4):223-8.

22. Rapoport A, Shaheed J, Newman C, Rugg M, Steele R. Parental perceptions of forgoing artificial nutrition and hydration during end-of-life care. Pediatrics. 2013;131(5):861-9. doi: 10.1542 /peds.2012-1916

23. Mahant S, Friedman JN, Connolly B, Goia C, Macarthur C. Tube feeding and quality of life in children with severe neurological impairment. Arch Dis Child. 2009;94(9):668-73. doi: 10.1136/adc.2008.149542

24. Druml C, Ballmer PE, Druml W, Oehmichen F, Shenkin A, Singer P, et al. ESPEN guideline on ethical aspects of artificial nutrition and hydration. Clin Nutr. 2016;35(3):545-56. doi: 10.1016/j.clnu.2016.02.006

25. Nelson LJ, Rushton CH, Cranford RE, Nelson RM, Glover JJ, Truog RD. Forgoing medically provided nutrition and hydration in pediatric patients. J Law Med Ethics. 1995;23(1):3346. doi: 10.1111/j.1748-720x.1995.tb01328.x

26. Zorzo RA, de Arruda F, da Costa JC. Nutrición en el paciente pediátrico en cuidados paliativos. Rev Nutr Clín Metabol. 2021;4(2). doi: 10.35454/rncm.v4n2.218

27. Weaver MS, Mooney-Doyle K, Kelly KP, Montgomery K, Newman AR, Fortney CA, et al. The benefits and burdens of pediatric palliative care and end-of-life research: A systematic review. J Palliat Med. 2019;22(8):915-26. doi: 10.1089/ jpm.2018.0483

28. Siden H. Pediatric palliative care for children with progressive non-malignant diseases. Children. 2018;5(2):28. doi: $10.3390 /$ children 5020028 